\title{
Variance Components for Body Weight in Japanese Quails (Coturnix japonica)
}

\section{Author(s)}

Resende RO

Martins EN

Georg PC

Paiva E

Conti ACM

Santos Al

Sakaguti ES

Murakami AE

Curso de Zootecnia

Maringá, PR, Brasil

\section{Mail Address}

Renata Oliveira Resende

Curso de Zootecnia

Rua Marquês de Abrantes, 831

87.020-170. Maringá, PR, Brasil

E-mail: renataresend@yahoo.com.br

\section{Keywords}

Bayesian inference, heritability, maternal environment.

\section{Acknowledgements}

The authors thank CNPq for financial support.

Arrived: september / 2004

Approved: january / 2005

\section{ABSTRACT}

The objective of this study was to estimate the variance components for body weight in Japanese quails by Bayesian procedures. The body weight at hatch (BWH) and at 7 (BW07), 14 (BW14), 21 (BW21) and 28 days of age (BW28) of 3,520 quails was recorded from August 2001 to June 2002. A multiple-trait animal model with additive genetic, maternal environment and residual effects was implemented by Gibbs sampling methodology. A single Gibbs sampling with 80,000 rounds was generated by the program MTGSAM (Multiple Trait Gibbs Sampling in Animal Model). Normal and inverted Wishart distributions were used as prior distributions for the random effects and the variance components, respectively. Variance components were estimated based on the 500 samples that were left after elimination of 30,000 rounds in the burn-in period and 100 rounds of each thinning interval. The posterior means of additive genetic variance components were $0.15 ; 4.18 ; 14.62 ; 27.18$ and 32.68; the posterior means of maternal environment variance components were $0.23 ; 1.29 ; 2.76 ; 4.12$ and 5.16 ; and the posterior means of residual variance components were $0.084 ; 6.43 ; 22.66 ; 31.21$ and 30.85 , at hatch, $7,14,21$ and 28 days old, respectively. The posterior means of heritability were $0.33 ; 0.35 ; 0.36 ; 0.43$ and 0.47 at hatch, 7 , 14,21 and 28 days old, respectively. These results indicate that heritability increased with age. On the other hand, after hatch there was a marked reduction in the maternal environment variance proportion of the phenotypic variance, whose estimates were $0.50 ; 0.11 ; 0.07 ; 0.07$ and 0.08 for BWH, BW07, BW14, BW21 and BW28, respectively. The genetic correlation between weights at different ages was high, except for those estimates between BWH and weight at other ages. Changes in body weight of quails can be efficiently achieved by selection.

\section{INTRODUCTION}

Many studies on poultry genetics are present in the scientific literature, especially those concerning improvements in chickens and laying hens. However, genetic researches about Japanese quails are rare.

Selection decisions should be based on the knowledge of the genetic structure of the population that has been selected to develop the quail lines, either for egg or meat production.

Heritability estimates for body weight at hatch, 7, 14, 21 and 28 days old in Japanese quails were reported to be $0.38 ; 0.12 ; 0.31 ; 0.12$ and 0.44 respectively, when Henderson methodology was used (Aggrey \& Cheng, 1994).

In Japanese quails, heritability values for body weight between 0.47 0.74 at 28 days old were reported by Minvielle (1998) in a review about animal improvement for production. 
Saatei et al. (2002) reported heritability estimates for body weight of $0.32 ; 0.20 ; 0.21 ; 0.20$ and 0.15 at hatch, 7, 14, 21 and 28 days old, respectively, in one to one sire and dam pedigree recorded Japanese quail.

Therefore, this study had as objective to estimate the variance components for body weight in Japanese quails by Bayesian procedures, using data recorded from August 2001 to June 2002.

\section{MATERIALS AND METHODS}

A hundred and twenty-five male and 375 female Japanese quails were used in Universidade Estadual de Maringá to establish a foundation population. During the experiment, animals were housed in individual cages. Mating at a sex ratio of 1 male to 3 females was begun at about 120 days of age. Eggs from each dam were stored separately at $15^{\circ} \mathrm{C}$ for 7 days before incubation. There were two storage periods and two incubations. Eggs were identified by sire and dam number before incubation. Incubated eggs were transferred to pedigree-identified hatching baskets 2 days prior to hatching. Quails were individually numbered after hatching.

Three thousand, five hundred and twenty quail chicks were obtained. They were weighed at hatch, $7,14,21$ and 28 days of age and sex was determined at 21 days of age by plumage color.

A multiple-trait animal model with additive genetic, maternal environment and residual effects was implemented by Gibbs sampling methodology. A single Gibbs sampling with 80,000 rounds was generated by the program MTGSAM (Multiple Trait Gibbs Sampling in Animal Model) (Van Tassel \& Van Vleck, 1995). Normal and inverted Wishart distributions were used as prior distributions for the random effects and the variance components, respectively. Variance components were estimated based on the 500 samples that were left after elimination of 30,000 rounds in the burn-in period and 100 rounds of each thinning interval.

\section{RESULTS AND DISCUSSION}

The posterior mean, credibility interval and high density region of the variance components (additive genetic, maternal environment and residual), at the 90\% level, at the different ages are presented in Table 1 . The variance components showed symmetrical posterior distribution. Overall heritability estimate, genetic correlation and phenotypic correlation are presented in Table 2.

\begin{tabular}{|c|c|c|c|c|}
\hline Variable & Age (days) & PM & $\mathrm{Cl}$ & HDR \\
\hline \multirow{5}{*}{$\sigma^{2 a}$} & 1 & 0.15 & $0.12-0.19$ & $0.12-0.19$ \\
\hline & 7 & 4.18 & $3.17-5.18$ & $3.17-5.17$ \\
\hline & 14 & 14.62 & $11.89-17.47$ & $11.88-17.41$ \\
\hline & 21 & 27.18 & $22.33-32.37$ & $22.15-32.01$ \\
\hline & 28 & 32.68 & $27.32-38.93$ & $27.13-38.46$ \\
\hline \multirow{5}{*}{$\sigma^{2} m$} & 1 & 0.23 & $0.20-0.26$ & $0.20-0.26$ \\
\hline & 7 & 1.29 & $0.95-1.67$ & $0.87-1.56$ \\
\hline & 14 & 2.76 & $1.90-3.72$ & $1.80-3.56$ \\
\hline & 21 & 4.12 & $2.70-5.61$ & $2.50-5.35$ \\
\hline & 28 & 5.16 & $3.55-6.85$ & $3.17-6.44$ \\
\hline \multirow{5}{*}{$\sigma^{2} e$} & 1 & 0.084 & $0.07-0.10$ & $0.07-0.10$ \\
\hline & 7 & 6.43 & $5.81-7.04$ & $5.91-7.10$ \\
\hline & 14 & 22.66 & $21.03-24.45$ & $21.02-24.44$ \\
\hline & 21 & 31.21 & $28.34-34.04$ & $28.35-34.04$ \\
\hline & 28 & 30.85 & $27.37-34.10$ & $27.82-34.41$ \\
\hline
\end{tabular}

\begin{tabular}{|c|c|c|c|c|c|}
\hline & BWH & BW07 & BW14 & BW21 & BW28 \\
\hline BWH & 0.33 & 0.20 & 0.10 & 0.04 & 0.10 \\
\hline BW07 & 0.32 & 0.35 & 0.90 & 0.84 & 0.71 \\
\hline BW14 & 0.26 & 0.72 & 0.36 & 0.94 & 0.87 \\
\hline BW21 & 0.21 & 0.66 & 0.77 & 0.44 & 0.93 \\
\hline BW28 & 0.22 & 0.57 & 0.73 & 0.80 & 0.48 \\
\hline
\end{tabular}

The heritability estimates disagree with the estimates reported by Aggrey \& Cheng (1994), probably because they employed the Henderson methodology, which analyzes the traits one by one. On the other hand, the present study used MTGSAM analysis, that evaluate the traits together, and therefore each trait contributes to the estimation of the others.

The heritability estimate for BW28 is within the values presented by Minvielle (1998).

Heritability increased with age. However, Saatei et al. (2002) reported that the heritability estimate reduced with age, probably because maternal environment and additive genetic effect were confused.

There is great influence of the environment effect on BWH. This effect reduces when age increases.

The genetic correlation between weights at different ages was high, except for the correlation between BWH and body weight at other ages. The fact that weight at hatch is limited by egg weight probably influenced this result.

These results indicate that the growth in quails can be improved by selection. 


\section{CONCLUSION}

These results indicate that heritability increases with age. On the other hand, there was a marked reduction in the proportion of the maternal environment variance of the phenotypic variance after hatch. The genetic correlation between weights at different ages was high, except for those estimates between BWH and body weight at other ages. It is suggested that body weight of quails can be efficiently improved by selection.

\section{REFERENCES}

Aggrey SE, Cheng KM. Animal model analysis of genetic (co)variances for growth traits in Japanese quail. Poultry Science 1994, 73:1822-1828.

Minvielle F. Genetics and breeding of Japanese quail for production around the world. In: Proceedings of the $6^{\circ}$ Asian Pacific Poultry Congress; 1998; Nagoya. Japan. p. 122-127.

Saatei M, Dewi IAp, Aksoy R, Kirmizibayrak T, Ulutas Z. Estimation of genetic parameter for weekly liveweight in one to one sire and dam pedigree recorded Japanese quail. In: $7^{\circ}$ World Congress on Genetic Applied to Livestock Production; 2002; Paris, France. p.20.

Van Tassel CP, Van Vleck LD. A manual for use of MTGSAM. A set for FORTRAN programs to apply Gibbs sampling to animal models for variance component estimation. Lincoln: Department of Agriculture, Agricultural Research Service; 1995. 86p. 